\title{
The Effects of Air and Helium on the Performance of the Ion Trap Detector When Used for Real-Time Monitoring of Some Atmospheric Pollutants
}

\author{
D. Cameron,* P. H. Hemberger, J. E. Alarid, and C. P. Leibman** \\ Chemical and Laser Sciences Division, Los Alamos National Laboratory, Los Alamos, New Mexico, USA
}

\section{J. D. Williams}

Department of Chemistry, Purdue University, West Lafayette, Indiana, USA

\begin{abstract}
The feasibility of using an ion trap detector for real-time monitoring of volatile organic compounds in atmospheric samples is demonstrated. Detection limits in the low part-permillion to low part-per-billion ranges are achieved with an operating dynamic range of at least 5 orders of magnitude. Sample introduction through semipermeable membranes and molecular leak valves was evaluated. The membrane introduction method provided detection limit enhancements of 10-300 times over the molecular leak method. The effects of partial pressure from air and helium buffer gas were studied, and it was determined that, for optimum amounts of air, the helium buffer gas had no apparent effect on sensitivity or resolution. Air acts as the buffer gas to enhance ion signals by collisionally damping ion motion. The enhancement of molecular ion abundance at high partial pressures of air is postulated to be due to charge exchange and possibly collisional stabilization reactions. ( $\mathrm{Am}$ Soc Mass Spectrom 1993, 4, 774-781)
\end{abstract}

$\mathrm{T}$ The need for sensitive real-time analyses has been documented in the areas of environmental monitoring [1-3], process monitoring, and process control [4-6] and has spurred the development of on-site and transportable instrumentation that is rugged, simple to operate, and relatively inexpensive [7-13]. Mass spectrometry, a well-established laboratory technique, has been used for on-site and field analyses, and commercial instrumentation is available for this purpose [14]. (Specific instruments include Mobile Environmental Monitor (MEM). Bruker Instruments; Manning Park, Billerica, MA; SpectraTrak. Viking Instruments. Reston, VA; and TAGA. Sciex, Inc. Thornhill, Ontario, Canada.) Previous work has demonstrated that a modified ion trap detector (ITD), coupled with a gas chromatograph (GC) $[7,8]$, and a modified ion trap mass spectrometer (ITMS) [13] can be used as field transportable instruments for determining volatile organic compounds in soil, water, and air matrices.

*Current address: Chemistry and Geochemistry Department, Montana Tech, Butte, Montana, USA.

**Current address: Environmental Management Division, Los Alamos National Laboratory, Los Alamos, New Mexico, USA.

Address reprint requests to Dr. D. Cameron, Chemistry and Geochernistry Department, Montana Tech, Butte, MT 59701.
The determination of organic pollutants in air is of prime importance because many volatile organic compounds have been implicated in the formation of ozone in the troposphere, in the depletion of the stratospheric ozone layer, and as potential human carcinogens [15-17]. Many methods of atmospheric analyses have relied on the collection of samples over time periods ranging from 1 to 24 hours, thereby producing timeaveraged data $[15,18-20]$. The need for improved temporal profiling on a real-time or short-time analysis period has been recognized $[19,20]$. Recent investigations have examined the use of atmospheric pressure ionization (API) with mass spectrometry and tandem mass spectrometry (MS/MS) for the direct sampling and real-time analysis of atmospheric pollutants [20-24]. A potential disadvantage of API is that the predominant ionization mechanism is both compoundand matrix-gas dependent. Therefore, the relative sensitivities of various analytes are highly dependent on the exact analysis conditions [22-25]. The use of an external glow-discharge ion source and the ITMS operating in the MS/MS mode appear to obviate the variable ionization problems [26]. A cheaper and simpler method, with concomitant sacrifices in specificity and sensitivity, is to sample air directly into an ITD, either through a conductance limiting orifice (i.e., leak valve) 
or through a semipermeable membrane to increase sensitivity [27]. Other studies using the ITMS or the Finnigan ITS-41 ion trap spectrometer (Finnigan MAT, San Jose, CA), with either direct sampling or a short preconcentration step and electron impact ionization or chemical ionization, have shown impressive sensitivities for many volatile organic compounds in air [28-31].

We describe the utility of the ITD operating in the electron impact ionization mode for the real-time analysis of airborne volatile organic compounds of environmental importance. Detection limits and linear dynamic ranges of the instrument are determined for several compounds using the exponential dilution method and permeation tubes. The effects of sample introduction through a molecular leak valve and a dimethyl silicone polymer membrane were evaluated. The effects of air pressure on signal intensity and mass resolution were also determined. It is postulated that, at high partial pressures of air, charge exchange is the dominant ionization mechanism.

\section{Experimental}

\section{Instrumentation}

A Finnigan MAT Model 705 ITD was used with the following modifications. The ITD manifold was fitted with a Varian model 0564-K2500-303 ionization gauge (Lexington, MA) coupled to a Granville-Phillips Series 20 gauge controller (Boulder, $\mathrm{CO}$ ) and an absolute pressure gauge MKS Instruments model 390HA-00001 Baratron capacitance manometer (Andover, MA). These devices were used to monitor sample and buffer gas pressures. Except as noted, all pressures are reported as absolute. The ITD was nperated without the Teflon ${ }^{\text {TM }}$ spacers between the ring and end cap electrodes. This was necessary to increase conductance during some of the studies at higher pressures. Electron ionization with the automatic gain control (AGC) option was used. The maximum ionization time was $24 \mathrm{~ms}$. The radiofrequency levels during the AGC prescan and normal mass scan are fixed at $20 \mathrm{Da}$ by the ITD; therefore, $\mathrm{H}_{3} \mathrm{O}^{+}$was excluded from the trap, but $\mathrm{N}_{2}^{+}$. and $\mathrm{O}_{2}^{+\cdot}$ from air were not. For one set of experiments the calibration compound vial was replaced with a vial containing trichloroethylene (TCE), which was degassed through three freeze-pump-thaw cycles. In this fashion a constant amount of $T C E$ could be admitted into the ion trap while variable amounts of air could be introduced through a leak valve.

\section{Exponential Dilution Experiments}

For the experiments using the exponential dilution apparatus, a $40 \mathrm{~L} / \mathrm{s}$ Balzers turbomolecular pump (Hudson, NH) was used to evacuate the ITD manifold. The GC transfer line was removed from the vacuum manifold and replaced with a $1.9-\mathrm{cm}$ stainless steel cross. A Vacumetrics (Ventura, CA) dimethyl silicone membrane separator was connected to the cross via a Granville-Phillips series 203 leak valve. A second Granville-Phillips leak valve was used for control of standards for calibration curves. The instrument is shown schematically in Figure 1. Helium buffer gas was introduced directly to the ion trap manifold to an uncorrected ion gauge pressure of $2.0 \times 10^{-5}$ torr. With the Teflon spacers removed as in these experiments, better sensitivity was obtained by introducing helium in this fashion as opposed to coaxial (with the sample) introduction. The capacitance manometer was not attached to the ITD manifold for these experiments. The ITD and the sample introduction manifold to the leak valves were heated to $200^{\circ} \mathrm{C}$ with heating tape. The ITD was scanned continuously at 1 scan/sec. Version 3.2 of the ITD software was used.

The exponential dilution method [32] was used for the dynamic calibration of the ITD. The exponential dilution apparatus consisted of an unheated $4670-\mathrm{mL}$

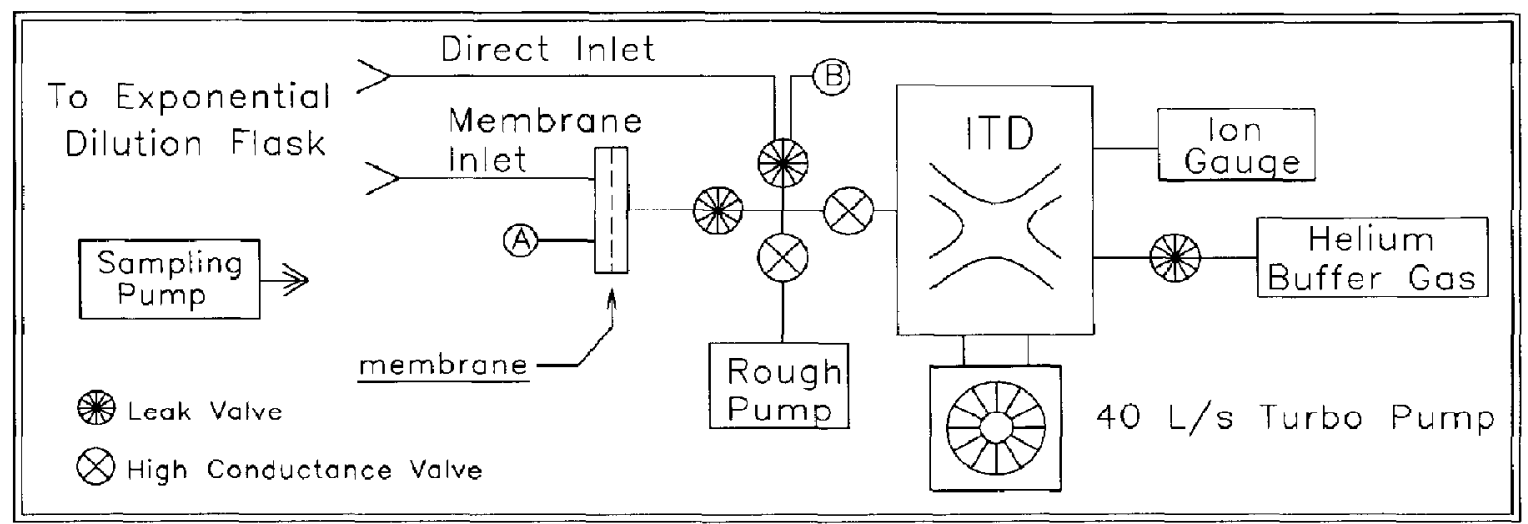

Figure 1. Schematic of ion trap and sample introduction system for exponential dilution experiments. The membrane inlet and leak valve were plumbed so that the gas stream from the exponential dilution flask impinged directly on the membrane or orifice, respectively. The sampling pump was connected to either point A or B for membrane or direct sampling. 
glass dilution flask containing a magnetically driven stirrer and an air-sampling pump. Air flow was regulated at $1000 \mathrm{~mL} / \mathrm{min}$ with a micrometer valve and measured with a calibrated Gilmont (Barrington, IL) flowmeter. If mixing is complete and the organic sample does not adsorb on the surfaces of the apparatus, the concentration of organic vapors $(C)$ decays with time according to eq 1 ,

$$
\mathrm{C}=\mathrm{C}_{0} \exp -(F t / V)
$$

where $V$ is the volume of the flask, $F$ is the flow rate, $C_{0}$ is the initial concentration, and $t$ is the elapsed time.

Initial organic vapor concentrations were 5000 and $500 \mathrm{ppm}$ (wt) in air for the leak valve and membrane separator, respectively. The concentrations were established by injecting a few microliters of the selected organic liquid into the dilution flask. Injection volumes were determined from the density of the organic liquid and the weight of air in the flask. Air density was calculated daily. Eppendorf digital pipettes (1-10 and $10-100 \mu \mathrm{L}$ ) were used for organic liquid delivery.

Gas was transferred from the dilution flask through unheated stainless steel tubing ( $3 \mathrm{~mm}$ o.d.) either to the leak valve or to the dimethyl silicone polymer membrane. The silicone membrane (Vacumetrics) was $0.076-\mathrm{mm}$ thick with an area of $3.8 \mathrm{~cm}^{2}$. An O-ring placed above the membrane (operated at $23{ }^{\circ} \mathrm{C}$ ) to reduce flow resistance increased the volume above the membrane to $0.5 \mathrm{~cm}^{3}$. Uncorrected sample pressures of approximately $4.5 \times 10^{-6}$ torr were obtained when the silicone membrane separator was used, and equivalent sample pressures were used when compounds were introduced through the leak valve.

Reagent-grade acetone and TCE were supplied by J. T. Baker Chemical Company (Phillipsburg, MA); reagent-grade benzene was supplied by Mallinckrodt (St. Louis, MO); and reagent-grade cyclohexane was obtained from Eastman Kodak Company (Rochester,
NY). All reagents were used as received without further purification.

\section{Permeation Tube Experiments}

For the experiments using permeation tubes, a $240 \mathrm{~L} / \mathrm{s}$ Balzers turbomolecular pump was used to evacuate the ITD manifold (Figure 2). The original Finnigan GC transfer line was replaced with a stainless steel line connected to a Granville-Phillips series 203 variable leak valve. The inlet side of the leak valve was plumbed with $6 \mathrm{~mm}$ o.d. stainless steel tubing connected to the dimethyl silicone membrane separator. The inlet line of the separator was connected by Teflon tubing to a VICI Metronics model 340-24-y Dynacalibrator (Santa Clara, CA). Helium buffer gas was introduced through a second leak valve for some experiments; the pressures used in those experiments are listed with the appropriate data in the Results and Discussion section. The ITD manifold was heated to $150{ }^{\circ} \mathrm{C}$. The leak valve and the stainless steel line from the leak valve to the ITD were heated to $50{ }^{\circ} \mathrm{C}$ to minimize background from sample adsorption. No background contamination from this source was observed at this temperature. Detection limit studies were done by continuously scanning the ITD at 2 scans $/ \mathrm{sec}$. The pressure studies were done in the manual tuning mode with Version 4.0 of the ITD software.

The VICI Metronics Dynacalibrator is designed to provide accurate and precise concentrations of gases in the parts-per-billion to high parts-per-million range. The operating principle of the instrument is based on the permeation of a gas at a constant rate through a containment device with controlled diffusion characteristics. Accurate control of the permeation tube temperature provides a precise amount of gas. Air is swept through the permeation chamber at a known flow rate to produce a gas mixture of predetermined concentration. The Dynacalibrator operating conditions specified by the manufacturer were used with the

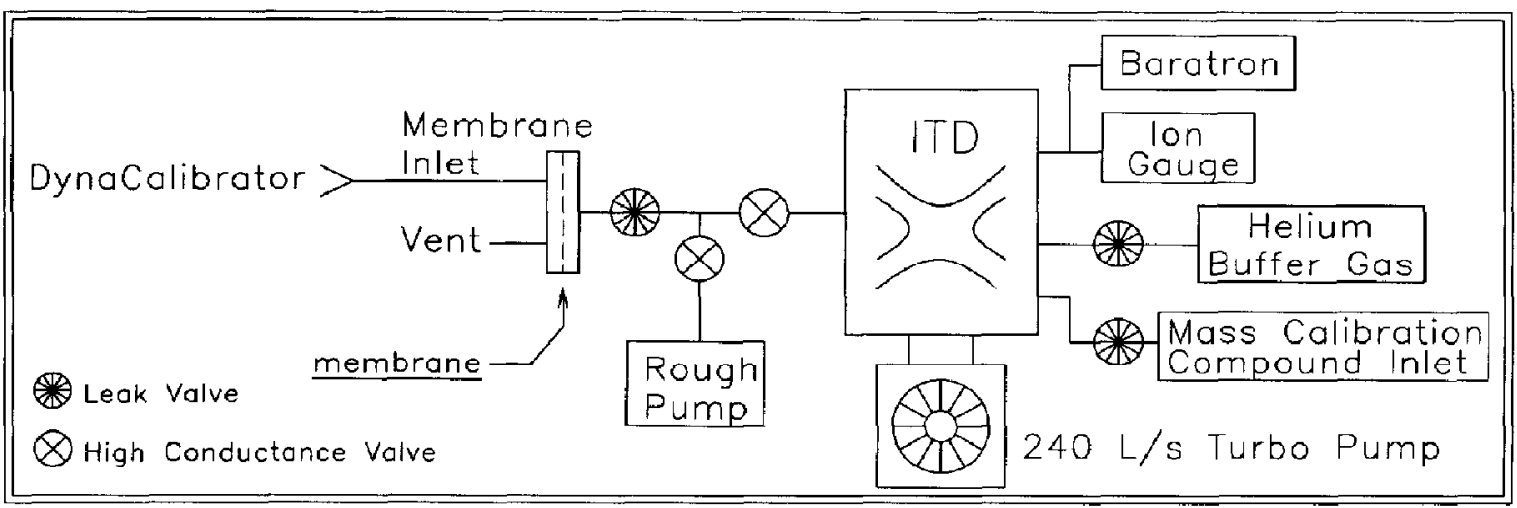

Figure 2. Schematic of ion trap and sample introduction system for permeation tube experiments. In one series of experiments, the membrane inlet was disconnected to allow introduction of air as a buffer gas, and TCE was introduced at the mass calibration compound inlet. 
appropriate corrections for ambient temperature and pressure. Because the Dynacalibrator is designed to provide the calibrated gas mixture at the current barometric pressure, constricting the Dynacalibrator chamber vent exhaust line was necessary to produce a gas flow to the membrane separator. The flow rate through the membrane separator vent line was $160 \mathrm{~mL} / \mathrm{min}$. Benzene and TCE were obtained from VICI Metronics in calibrated Teflon permeation tubes.

\section{Results and Discussion}

The exponential dilution method for gas mixture preparation was used for the initial series of experiments. Table 1 lists the compounds studied, the ions used for quantitation, and the detection limits using molecular leak and membrane sample introduction. The detection limits were determined when the ion signal monitored was three times the noise signal $(\mathrm{S} / \mathrm{N}=3 / 1)$. Enhancement of the detection limits for the organic component in the gas mixtures sampled through the dimethyl silicone membrane is indicated by the data in Table 1 and graphically in Figure 3. Figure 3 is a log-log plot of the measured signal intensity versus organic vapor concentration (ppm-wt). Signal enhancement results mainly from the enrichment of the organic compounds relative to the normal constituents of air [33]. Minor secondary effects may occur due to slight differences in gas composilion or pressure. The data points in Figure 3 were obtained by plotting observed signal intensities versus the calculated concentrations in the dilution flask. Each point reflects the mean intensity and the median concentration of 17 scans. The linearity of the plots in Figure 3 indicates the minimum dynamic range of the analysis is approximately 5 orders of magnitude when using the membrane sampler. The upper concentration range of the technique has not yet been investigated. The linearity of the data in Figure 3 also indicates that the exponential dilution method worked from the highest vapor concentrations established $(5000 \mathrm{ppm}$ for the leak valve sampling and $500 \mathrm{ppm}$ for the membrane sampling) to the noise limit. The differences in sensitivities among TCE, acetone, and benzene for the direct introduction data can be ascribed to differences in their vapor pressure, whereas the enhanced response to benzene in the membrane data is likely due to benzene's higher transport through the silicone mem-

Table 1. Detection Limits for Trace Organic Vapors in Air with Leak Valve and Semipermeable Membrane Sampling

\begin{tabular}{lccc}
\hline & \multirow{2}{*}{$\begin{array}{c}\text { Mass-to-charge } \\
\text { Compound }\end{array}$} & \multicolumn{2}{c}{ Limits of Detection } \\
\cline { 3 - 4 } \multicolumn{1}{c}{ ratio monitored } & Leak valve & Membrane \\
\hline \hline Benzene & 78 & $1 \mathrm{ppm}$ & $3 \mathrm{ppb}$ \\
Trichloroethylene & 130 & $8.3 \mathrm{ppm}$ & $32 \mathrm{ppb}$ \\
Acetone & 43 & $39 \mathrm{ppm}$ & $2.8 \mathrm{ppm}$ \\
Cyclohexane & 84 & $20 \mathrm{ppm}$ & $640 \mathrm{ppb}$ \\
\hline
\end{tabular}

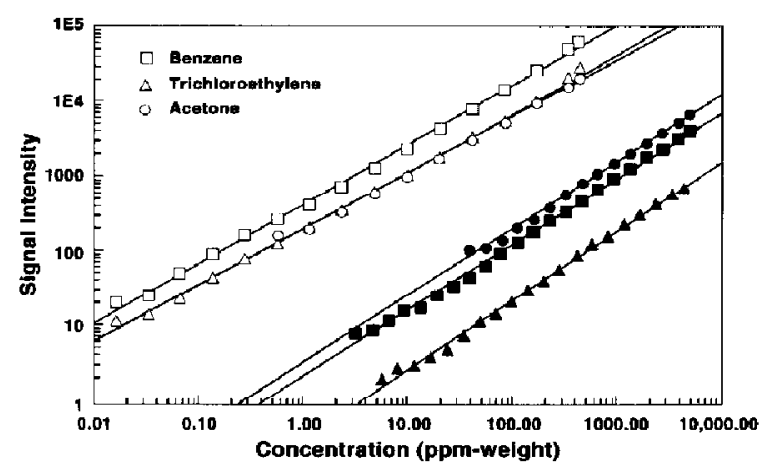

Figure 3. Log-log plot of signal intensity versus concentration for benzene, TCE, and acetone in air. Open symbols indicate compounds sampled through the semipermeable membrane. Closed symbols indicate compounds sampled directly.

brane. It is likely that the detection limits reported here could be improved by deactivating the glassware in the exponential dilution flask and transfer line [34].

Silicone membrane separators employed as an interface between a $G C$ and a mass spectrometer are operated at elevated temperatures to maintain chromatographic resolution. However, permeation of organic vapors decreases with increasing membrane temperature [35, 36]. Therefore, the silicone membrane was operated at room temperature to enhance the permeation of the organic vapors relative to the inorganic atmospheric gases. Operation of the membrane at room temperature did not pose a problem in the exponential dilution experiments reported here because the instrument responded rapidly to abrupt changes in organic vapor concentration. The molecular ion signal from TCE decayed to $5 \%$ of the maximum signal level within $18 \mathrm{sec}$ from the time the transfer line was removed from the dilution flask. Shorter decay times could have been achieved if the transfer line were disconnected at the membrane; however, this was not possible with the experimental set-up.

The permeation tubes were initially used to confirm the detection limits from the exponential dilution experiments. However, the ease of operation and the stable output of gas mixtures from the permeation tubes permitted the characterization of other experimental parameters. The first of these parameters is the membrane separator. We have investigated only dimethyl silicone membranes, but the enhancement of organic vapors relative to the atmospheric gases is dependent on the membrane material. Improvements in membrane selectivity to increase the transference of organics relative to air could lead to lower detection limits and, as discussed below, increase the longevity of the ionization filament and electron multiplier.

The manufacturer-supplied separator and membrane, as used in the sensitivity experiments described above, allowed limited transfer of gaseous material into the ion trap. Maximum pressures of approximately $0.5-1 \times 10^{-5}$ torr were obtained in the ITD 
when the membrane assembly was used as received. By using replacement membranes, the pressure in the trap could easily exceed $1 \times 10^{3}$ torr. The replacement membranes were sealed to the separator assembly with a silicone epoxy according to the manufacturer's instructions. Inspection of the seal and the membrane revealed no visible tears or holes. We believe that some channels through or around the membrane were produced during the replacement process. In addition to the higher manifold pressures, the replacement membranes produced slightly lower enrichment factors, which is consistent with some air leaking directly into the ITD through or around the membrane assembly. The studies on the operating effects of pressure described below were performed with the increased pressure range available with the replacement membrane.

The second parameter evaluated for the operation of the ITD was the partial pressure of helium and air. TCE was used to examine the effects of air and helium partial pressures on the sensitivity and the mass resolution of the ITD. TCE was emitted from the Dynacalibrator at a roncentration of $1.1 \mathrm{ppm}$ (wt) in air. The pressure of helium admitted into the ITD was measured with the Baratron manometer. Figure 4 plots the signal intensities versus air pressure at three different helium pressures. To reduce the random fluctuation in instrument response, the plotted signal intensity is the average of the intensities of the $\mathrm{m} / \mathrm{z} 130$ and 132 isotope molecular ions. The increase in the ion signal intensity as a function of air pressure in Figure 4 is the result of several factors:

- Increasing amounts of TCE are admitted into the ion trap as mure air is introduced because the concentration of TCE in air is fixed.

- The air acts as a buffer gas, in a manner similar to helium, enhancing the sensitivity by collisional focusing $[37,38]$.

- The ionization mechanism changes from electron ionization to predominantly charge exchange as the air pressure increases [39].

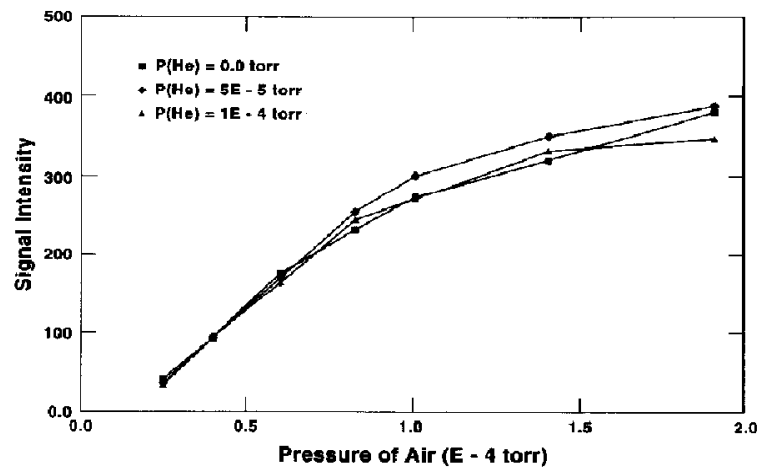

Figure 4. Signal intensity of TCE versus pressure of air at three different partial pressures of helium.
- Collisional stabilization of the molecular ion may be occurring by the interactions of TCE with the constituents of air [40].

At these high partial pressures of air (0.5-2 $\times$ $10^{-4}$ torr), supplemental helium as a buffer gas has no apparent effect on signal intensity. The results at helium partial pressures higher than those shown in Figure 4 are similar except for a large increase in base-line noise at the higher pressures. The signal enhancement caused solely by the buffer gas (air) is observed in the lower trace of Figure 5. These data are from an experiment where the partial pressure of TCE, introduced to the ion trap via the mass calibration compound inlet, was constant and the amount of air was varied. Although this result may seem contrary to previous work showing the effects of helium buffer gas [37], our data indicate that air may effectively serve as a buffer gas in the ITD. The effectiveness of air as a buffer gas in the ITD may be abetted by the presence of higher-order (octapole) fields. The Finnigan ion trap has a "stretched" geometry in which the end electrodes are symmetrically displaced from the center of the trap such that the distance from the center of the trap to each electrode $\left(z_{0}\right)$ is $7.83 \mathrm{~mm}$ [41]. In a quadrupole ion trap having theoretically correct geometry with a ring electrode of radius $r_{0}=$ $10.00 \mathrm{~mm}, z_{0}$ would equal $7.07 \mathrm{~mm}$. This symmetrical displacement of the end electrodes adds an octapole component to the quadrupole field potential [42]. It has been shown that ion traps which have a weak octapole field superimposed on the quadrupole field are capable of long storage times (hours) using air as the buffer gas [43]. Our data indicate that air can serve as a buffer gas in the ITD for ions of moderately low mass-to-charge ratios (approximately $150 \mathrm{Da}$ ), which includes most of the volatile organic compounds of environmental significance.

The partial pressure of air in the ion trap also affects the relative abundance of the molecular ions and fragment ions. This effect is seen in Figure 6 where the ratio of molecular to fragment ion signal intensities is plotted versus the pressure of air in the trap for three different fragment ions from TCE. The ions chosen are all monoisotopic in ${ }^{35} \mathrm{Cl}$. The data show an increase in

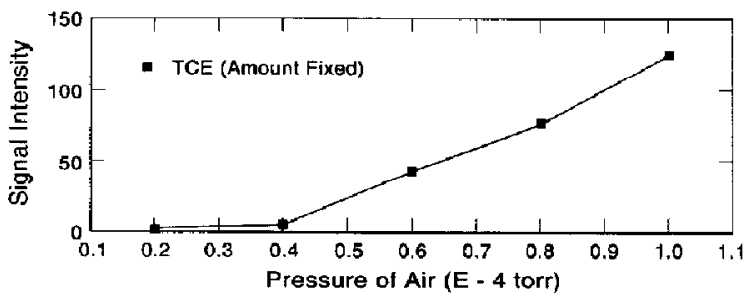

Figure 5. Signal intensity for TCE versus pressure of air. Signal intensities are averages of the $m / z \quad 130$ and 132 molecular ions for a constant amount of TCE in the ion trap. The data show that air acts as a buffer gas. 


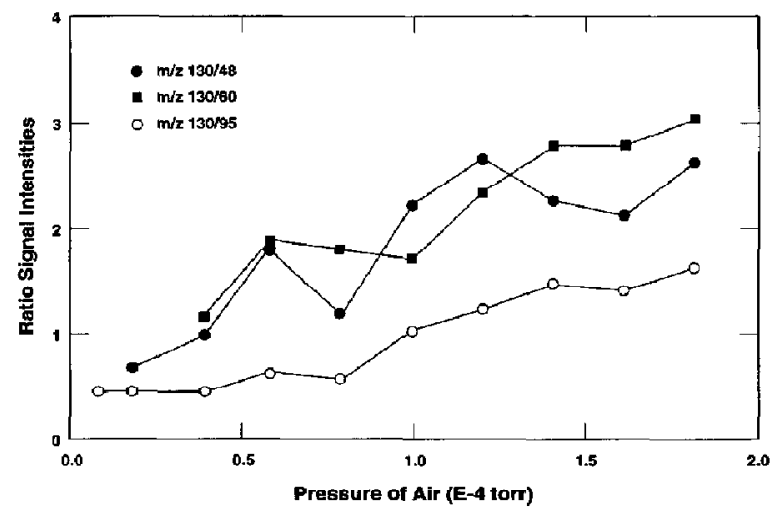

Figure 6. Ratio of molecular ion to fragment ion signal intensities from TCE versus pressure of air. Enhancement of relative molecular ion abundance is observed with increasing air pressure-

the relative amount of the molecular ion $m / z 130$ with increasing air pressure. Minor differences, which may be due to the energetics of the various fragmentation reactions, are noted in the slopes of the plots. At the lower air pressures, ratios are not reported for some of the fragment ions because these ions were not detected. The enhancement of the molecular ion to the fragment ions is thought to be the result of two factors: a different ionization mechanism and collisional stabilization. Although not plotted in Figure 6, the absolute intensity of all ions shown in that figure increases with pressure over the given range, which suggests that the observed trend is not due to loss of the lower molecular weight ions through scallering. Previous studies [39] have reported that, in the pressure range of these studies $\left(1 \times 10^{-6}\right.$ to $1 \times 10^{-4}$ torr of air), the predominant ionization mechanism changes from electron ionization to charge exchange as the pressure is increased. Although charge exchange between $\mathrm{O}_{2}^{+-}$or $\mathrm{N}_{2}^{+-}$and TCE is relatively energetic $(2.7 \mathrm{eV}$ and $6.3 \mathrm{eV}$, respectively), the energy imparted to TCE by charge exchange ionization with $\mathrm{O}_{2}^{++}$is less than the upper range of internal energies produced by electron ionization [44]. Although charge exchange by $\mathrm{N}_{2}^{+-}$might lead to an average internal energy higher than that found by electron ionization* [45, 46], some of our work [39] indicates that at the air pressures used here, $\mathrm{O}_{2}^{+-}$is the major ion species in the ITD. The intensity ratio $32 / 28$ for $\mathrm{N}_{2}^{+-}$and $\mathrm{O}_{2}^{+-}$increases with increasing air pressure in the ITD. This ratio eventually stabilizes at a value of approximately 2 . We therefore expect an increase in the relative amount of molecular ion, formed by charge exchange with $\mathrm{O}_{2}^{+}$, with increasing air pressure. It is not possible to determine from these data if collisional stabilization of the TCE molecular ions was occurring, although it is reasonable to assume that collisional stabilization reactions do occur under the multiple collision conditions of the ion trap. The relative effi-

${ }^{*} N_{2}$ charge exchange spectrum of TCE contains an intense peak at $m / z 35$ and only minor peaks at $m / z 130$ and 132 . ciency of various collisional stabilization gases has been reported for specific ionic systems [40]. From these data we expect the deactivation effects of air to be noticeable when compared to helium. Further study on bath gas effects in the ion trap certainly are needed.

The $\mathrm{m} / \mathrm{z} 129$ fragment ion, not plotted in Figure 6, did not show the same pressure effects as the other fragment ions from $m / z$ 130. This anomalous behavior is an artifact of the experiment. At low pressures and low amounts of TCE in the trap, the $m / z 129$ ion is not detected. Only at high air pressures or with large amounts of TCE can a reliable determination of the abundance of the $m / z 129$ ion be obtained. In experiments where these criteria are met, the pressure profile of the $m / z 129$ ion tracks those of the other fragment ions.

The effects of the partial pressure of helium and air on the mass resolution are shown in Figures 7 and 8 . Resolution is defined here as the peak width at either $50 \%$ or $5 \%$ of maximum peak height (Figures 7 and 8 , respectively) divided by peak separation $(\Delta m)$. These definitions are admittedly not the traditional ones for resolution; this method is simply a convenient way to look at peak broadening using the ITD software. The peaks from the molecular ions $\mathrm{m} / z 130$ and 132 were used for these determinations, and the averages for these two peaks are used in Figures 7 and 8 . The data in Figure 7 indicate a monotonic decline in resolution (50\% peak height) with increasing gas pressure. The data in Figure 8 show a plateau for resolution (5\% peak height) until approximately $1 \times 10^{-5}$ torr, followed by a sharp decrease in resolution with increasing pressure. (At air pressures less than $2 \times 10^{-5}$ torr, signal levels were too low for accurate peak width measurements.) The presence of helium as a buffer gas at these pressures appears to have little or no effect on the mass resolution in the ion trap. The mass resolution data at $5 \%$ peak height indicate substantial peak broadening with increasing air pressure, which suggests that space charging is becoming a limiting factor at these pressures, although collisional broadening may

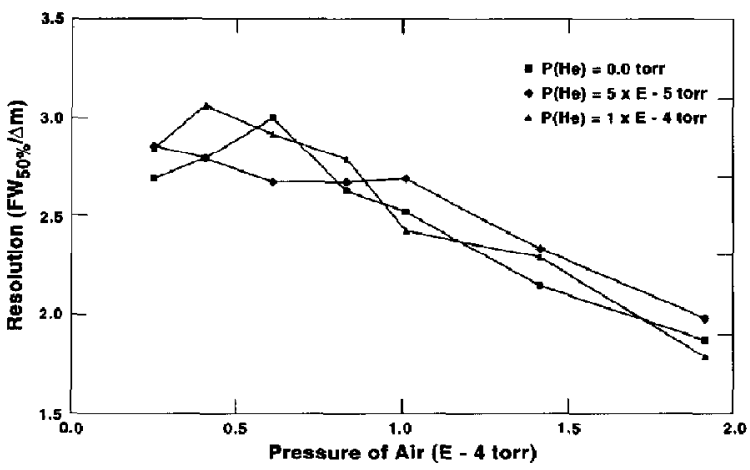

Figure 7. Resolution ( $50 \%$ peak height) versus pressure of air at three partial pressures of helium. Resolution is the average value for $m / z 130$ and 132 from TCE. The resolution decreases with increasing air pressure, and the helium appears to have no effect. 


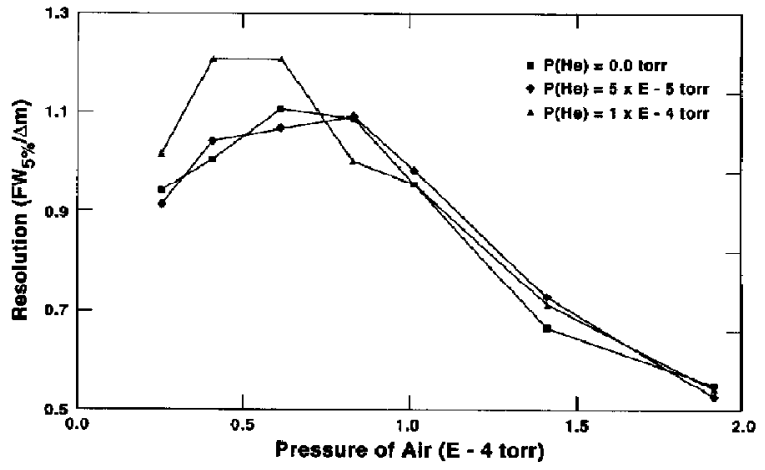

Figure 8. Resolution (5\% pcak height) versus pressure of air at three partial pressures of helium. Resolution is the average value for $m / z 130$ and 132 from TCE. The resolution decreases with increasing air pressure, and the helium appears to have no effect.

also be occurring. Comparing the results in Figures 4 and 8 suggests that a maximum pressure of $1 \times 10^{-4}$ is a good compromise between signal intensity and mass resolution. Although improved sensitivity is found with the higher air pressures in the ion trap, the increased partial pressure of oxygen substantially reduces the lifetime of the ionization filament and the electron multiplier. Under the conditions of these experiments, filament lifetimes were about 3 weeks with 2 to 4 hours daily operation.

\section{Conclusions}

The ITD, coupled with semipermeable membrane sample introduction, has the sensitivity and dynamic range for real-time munitoring of organic atmospheric pollutants. The partial pressure of air in the trap affects the sensitivity and mass resolution of the technique and the relative abundance of molecular and fragment ions. With air partial pressures of $5 \times 10^{-6}$ torr and greater, helium buffer gas has no apparent beneficial effect for the system studied. At partial pressures of air greater than $1 \times 10^{-5}$ torr, molecular ion abundance is enhanced relative to fragment ion abundance. One explanation is that air, notably $\mathrm{O}_{2}^{+}$, acts as a charge exchange reagent and alters the ionization mechanism. Collisional stabilization reactions may also be occurring at these pressures. The presence of large partial pressures of oxygen severely decreases the useful lifetime of the ionization filament and the electron multiplier. The development and application of selective membranes to enhance the ratio of organic vapors to oxygen would be extremely beneficial.

Previous work has amply demonstrated the use of semipermeable membranes with either quadrupole or ITMS for the on-line analysis of organic contaminants in aqueous and nonaqueous media $[47,48]$. We demonstrate here that the ion trap detector is capable of real-time monitoring of atmospheric pollutants with a simple and inexpensive modification, that is, a mem- brane sampling interface. Although further work is needed to fully assess the strengths and weaknesses of the ITD in this application, the speed of analysis (near real-time), sensitivity (parts-per-billion), and simplicity of the instrumentation make this an attractive methodology for air monitoring.

\section{References}

1. Cram, S. P. Amer. Environ. Lab. 1989, 1, 19-26.

2. Chudyk, W. Environ. Sci. Technol. 1989, 23, 504-507.

3. Daley, P. S. Environ. Sci. Technol. 1989, 23, 912-916.

4. Callis, J. B.; Illman, D. L.; Kowalski, B. R. Anal. Chem. 1987, 59, 624A-637A.

5. Symposium: "The Analytical Chemistry Opportunity in Process Instrumentation." Pittsburgh Conference, Atlantic City, NJ, March 1987.

6. Riebe, M. T.; Eustace, D. J. Anal. Chem. 1990, 62, 65A-71A.

7. Leibman, C. P.; Cannon, T. M.; Wolf, M. A.; Hemberger, P. H. Procedings of the 37th Annual ASMS Conference on Mass Spectrometry and Allied Topics, Miami Beach, FL, May 1989; pp $1429-1430$.

8. Hemberger, P. H.; Alarid, J. E.; Cameron, D.; Cannon, T. M.; Kaiser, R. E.: Leibman, C. P.; Wolf, M. A. Int. I. Mass Spectrom. Ion Proc. 1991, 106, 299-313.

9. McClenny, W. A,; Oliver, K. D; Piell, J. D. Environ. Sci. Technol. 1989, 23, 1373-1379.

10. Blanchard, R. D.; Hardy, J. K. Anal. Chem. 1986, 58, 15291532.

11. Spartz, M. L.; Witkowski, M. R.; Fateley, J. H.; Jarvis, J. M.; White, J. S.; Paukstelis, J. V.; Hammaker, R. M.; Fateley, W. G.; Carter, R. E.; Thomas, M.; Lane, D. D.; Marotz, G. A.; Fairless, B. J.; Holloway, T.; Hudson, J. L.; Gurka, D. F. Amer. Environ. Lab. 1989, 1, 15-30.

12. Grupp, D. J.; Everitt, D. A.; Bath, R. J.; Spear, R. Amer, Environ. Lab. 1989, 1, 32-40.

13. Arnold, N. S.; Roberts, K. A.; McClennen, W. H.; Meuzelaar, H. L. C. Proceedings of the 37th Annual ASMS Conference on Mass Spectrometry and Allied Topics, Miami Beach, FL, May 1989; pp 1425-1426.

14. Cannon, D. R. Ind. Chem. News 1986, 7, 1 .

15. Shah, J. J.; Singh, H. B. Environ. Sci. Technol. 1988, 22, 13811388

16. Chock, D. P.; Heuss, J. M. Enviran. Sci. Technol. 1987, 21 1146-1153.

17. Cohen, Y. Environ. Sci. Technol. 1986, 20, 538-544.

18. Williams, E. J.; Sievers, R. E. Anal. Chem. 1984, 56, 2523-2528.

19. McClenny, W. A.; Pleil, J. D.; Holdren, M. W.; Smith, R. A. Anal. Chem. 1984, 56, 2947-2951.

20. MoClenny, W. A.; Oliver, K. D.; Pleil, J. D. Environ. Sci. Technol. 1989, 23, 1373-1379.

21. Asano, K. G.; Glish, G. L.; McLuckey, S. A. Proceedings of the $36 t h$ Annual ASMS Conference on Mass Spectrometry and Allied Topics, San Francisco, CA, June 1988; pp 636-637.

22. McLuckey, S. A.; Glish, G. L.; Asano, K. G.; Grant, B. C Anal. Chem. 1988, 60, 2220-2227.

23. Sunner, J.; Nicol, G.; Kebarle, P. Anal. Chem. 1988, 60, 13001307 .

24. Sunner, J.; Ikonomou, M. G.; Kebarle, P. Anal. Chen. 1988, $60,1308-1313$.

25. Ketkar, S. N.; Dulak, J. G.; Fite, W. L.; Buchner, J. D.; Dheandhanoo, S. Anal. Chem. 1989, 61, 260-264.

26. Eckenrode, B. A.; Glish, G. L.; McLuckey, S. A. Proceedings of the 37th Annual ASMS Conference on Mass Spectrometry and Allied Topics, Miami Beach, FL, May 1989; pp 462-463. 
27. Hemberger, P. H.; Spall, W. D; Leibman, C. P.; Cannon, T. M. Proceedings of the 36th Antrual ASMS Conference on Mass Spectrometry and Altied Topics, San Francisco, CA, June 1988; $\mathrm{p} 638$.

28. Wise, M. B.; Buchanan, M. V.; Ilgner, R. H. Proceedings of the 37th Annual ASMS Conference on Mass Spectrometry and Allied Topics, Miami Beach, FL, May 1989; p 1435.

29. Wise, M. B.; Ilgner, R. H.; Buchanan, M. V. Proceedings of the 38th Annual ASMS Conference on Mass Spectrometry and Allied Topics, Tucson, AZ, June 1990; $\mathrm{p} 1481$.

30. Berberich, D. W.; Orth, R. G. Proceedings of the 39th Annual ASMS Conference on Mass Spectrometry and Allied Topics, Nashville, TN, May 1991; p 1279.

31. Thompson, C. V.; Wise, M. B.; Blalock, A. V.; Hurst, G. A. Proceedings of the 40th Annual ASMS Conference on Mass Spectrometry and Allied Topics, Washington, DC, June 1992; p 653.

32. Lovelock, J. E. Anal. Chem. 1961, 33, 1632-178.

33. Matz, G.; Trinks, H. Int. J. Mass Spectrom. Ion Physs, 1982, 43, 79-82.

34. Glish, G. L.; McLuckey, S. A, Personal communication.

35. Grayson, M. A.; Wolf, C. H. Anal. Chem. 1970, 42, 426-430.

36. Greenwalt, C. C.; Voorhees, K. J.; Futrell, J. H. Anal. Chert. $1983,55,468-472$.

37. Stafford, Jr. G. C.; Kelley, P. E.; Syka, J. E. P.; Reynolds, W. E.; Todd, J. F. J. Int. J. Mass Spectrom. Ion Proc. 1984, 60, 85-98.
38. Hemberger, P. H.; Nogar, N. S.; Williams, J. D.; Cooks, R. G.; Syka, J. E. P. Chem. Phys. Lett. 1992, 191, 405-410.

39. Cameron, D.; I Iemberger, P. II. Proceedings of the 38th Annual ASMS Conference on Mass Spectrometry and Allied Topics, Tucson, AZ, June 1990; pp 61-62.

40. Lambert, J. D. Vibrational and Rotational Relaxation in Gases; Oxford University: Oxford, 1977.

41. Louris, J.; Schwarz, J.; Stafford, G.; Syka, J.; Taylor, D. Proceedings of the 40th Annual ASMS Conference on Mass Spectrometry and Allied Topics, Washington, DC, June 1992; pp 1003-1004.

42. Dawson, P. H.; Whetten, N. R. Int. J. Mass Spectrom. Ion Phys. $1969,2,45-59$.

43. Franzen, J.: Gabling, R.-H. Proceedings of the 40th Annual ASMS Conference on Mass Spectrometry and Allied Topics, Washington, DC, June 1992; pp 1009-1010.

44. McLafferty, F. W. Interpretation of Mass Spectra; University Science Books: Mill Valley, California, 1980; Chapter 7.

45. Strife, R. D. Personal communication.

46. Berberich, D. W.; Hail, M. E.; Johnson, J. V.; Yost, R. A. Int. I. Mass Spectrom. Ion Phys. 1989, 94, 115-147.

47. Cooks, R. G.; Kotiaho, T. ACS Symposium Series, 1992, 508, 126-154.

48. Kotiaho, T.; Lauritsen, F. R.; Choudhury, T. K.; Cooks, R. G.; Tsao, G. T. Anal. Chem. 1991. 63, 875A. 